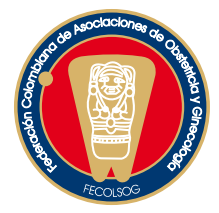

\title{
COMPLICACIONES LAPAROSCÓPICAS ASOCIADAS A LA TÉCNICA DE ENTRADA
}

\section{Entry technique-associated laparoscopic complications}

Janer Sepúlveda-Agudelo, M.D.*

Recibido: febrero 5/10 - Aceptado: enero 17/11

\section{RESUMEN}

Objetivo: realizar una revisión detallada de las complicaciones de la cirugía laparoscópica en ginecología, con énfasis en las relacionadas con la entrada a la cavidad peritoneal dada su alta frecuencia.

Materiales y métodos: se realizó una búsqueda detallada de literatura publicada en inglés a través de PubMed/MEDLINE, Ovid y registro de revisiones sistemáticas y estudios aleatorizados controlados de Cochrane, usando como palabras clave "laparoscopic entry", "trocars injury", "laparoscopy complications", "laparoscopic injury" y "optical trocars". Se seleccionaron principalmente metaanálisis, estudios clínicos aleatorizados, guías clínicas, artículos de revisión y series de casos.

Resultados: se seleccionaron 36 artículos, 2 metaanálisis, 3 estudios clínicos aleatorizados, 1 guía clínica, 11 revisiones sistemáticas, 10 revisiones de tema y 9 series de casos. Las complicaciones de la cirugía laparoscópica son raras, se presentan con mayor frecuencia durante la entrada y generalmente son de origen vascular, intestinal y urológico. Igualmente, pueden presentarse complicaciones por el neumoperitoneo y por electrocirugía. Otras complicaciones que ocurren con menor frecuencia son la neurológicas, las hernias incisionales, las

* Médico Gineco-obstetra, Especialista en Cirugía Endoscópica Ginecológica. Profesor Asistente, Departamento de Gineco-obstetricia, Universidad Industrial de Santander. Miembro, grupo de investigación GINO. Bucaramanga (Colombia).

Correo electrónico: janersepulveda@yahoo.es metástasis por el sitio de entrada, las infecciones, los hematomas y la formación de adherencias.

Conclusiones: la laparoscopia es un procedimiento relativamente seguro. Sin embargo, hay que ser muy cuidadoso en el momento del acceso a la cavidad abdominal, porque la mayoría de complicaciones ocurren durante la entrada.

Palabras clave: entrada laparoscópica, lesiones con trócares, complicaciones laparoscópicas, lesiones laparoscópicas, trócares ópticos.

\section{SUMMARY}

Objective: carrying out a detailed review of complications arising from laparoscopic surgery used in gynecology, emphasizing those related to entering the peritoneal cavity as being the most frequently presented.

Materials and methods: a detailed search was made of literature published in English in PubMed/ MEDLINE, Ovid and the Cochrane database of systematic reviews and randomized controlled trials using the following key words: "laparoscopic entry", "trocars injury", "laparoscopy complications", "laparoscopic injury" and "optical trocars". Metanalysis, randomized clinical studies, clinical guidelines, review articles and case series were mainly selected.

Results: 36 articles, 2 metanalyses, 3 randomized clinical studies, 1 clinical guide, 11 systematic reviews, 10 topic reviews and 9 case series were selected. Complications rarely arise during laparoscopic surgery; they occur most frequently during entry and are generally 
of vascular, intestinal or urological origin. Complications may likewise be presented by pneumoperitoneum and electrosurgery. Less frequently occurring complications are neurological in origin or involve incisional hernias, metastasis at the entry site, infections, hematomas and adherence formation.

Conclusions: laparoscopy represents a relatively safe procedure; however, great care must be taken when gaining access to the abdominal cavity as most complications occur during such entry.

Key words: laparoscopic entry, trocar lesions, laparoscopic complications, laparoscopic lesions, optical trocar.

\section{INTRODUCCIÓN}

El uso de laparoscopia para la realización de procedimientos en ginecología va en aumento puesto que proporciona mayores beneficios, cuando se compara con la laparotomía, en cuanto a una recuperación más rápida, menor dolor operatorio y estadía hospitalaria más corta. ${ }^{1}$ Sin embargo, dado que es un procedimiento invasivo existe el riesgo de que surjan complicaciones. ${ }^{2}$ Dichas complicaciones ocurren especialmente al momento del ingreso de los instrumentos endoscópicos a la cavidad abdominal. ${ }^{3,4}$ Las complicaciones de la cirugía ginecológica laparoscópica son raras, con una incidencia de 3 a 6 por cada 1.000 casos y la tasa de mortalidad está estimada en 3,3 por cada 100.000 procedimientos. ${ }^{5}$ Estas aumentan directamente con el nivel de complejidad de los procedimientos y disminuyen con la experiencia del cirujano. ${ }^{5,6}$ Sin embargo, estas complicaciones no son mayores en comparación con la laparotomía. Chapron y colaboradores no encontraron diferencias entre las complicaciones mayores entre pacientes manejadas con laparoscopia o con laparotomía para patología ginecológica benigna, aunque sí encontraron un riesgo más bajo de complicaciones menores con la laparoscopia. ${ }^{7}$

El objetivo de este documento es realizar una revisión detallada de las complicaciones de la cirugía laparoscópica en ginecología. Puesto que son las más frecuentes, se hace énfasis en aquellas relacionadas con las técnicas de entrada a la cavidad.

\section{MATERIALES Y MÉTODOS}

Se realizó una búsqueda de literatura publicada en inglés a través de las bases de datos PubMed/ MEDLINE, Ovid y el registro de revisiones sistemáticas y estudios aleatorizados controlados de la colaboración Cochrane, usando los términos: "laparoscopic entry", "trocars injury", "laparoscopy complications", "laparoscopic injury", y "optical trocars". Se seleccionaron revisiones sistemáticas y narrativas, guías clínicas, estudios clínicos aleatorizados y series de casos.

\section{RESULTADOS}

Se revisaron 36 artículos: 2 metaanálisis, 11 revisiones sistemáticas cualitativas, 1 guía clínica, 3 estudios clínicos aleatorizados, 10 revisiones de tema y 9 series de casos.

\section{Complicaciones mayores Lesiones vasculares}

Tienen una incidencia de $0,01 \%$ a $0,64 \%,{ }^{5}$ pero son severas con una tasa de mortalidad del $9 \%$ y $17 \% .^{8}$ Anatómicamente, los vasos de mayor riesgo durante la entrada son la arteria aorta, la vena cava inferior y los vasos iliacos. ${ }^{5}$ Se deben sospechar ante evidencia rápida intraoperatoria de inestabilidad hemodinámica o la presencia de un hematoma retroperitoneal. ${ }^{9}$ Los vasos sanguíneos en la pared abdominal también pueden resultar comprometidos durante el ingreso, sus lesiones son reportadas con una frecuencia de $0,2 \%$ a $2 \% .{ }^{10}$ El vaso que más se ve comprometido es la arteria epigástrica inferior, y esto ocurre generalmente por inserción de trócares laterales. ${ }^{11}$

\section{Lesiones intestinales}

Son la causa de más de la mitad de las complicaciones mayores en cirugía laparoscópica con una incidencia de $0,06 \%$ a $0,65 \% ;^{5}$ estas lesiones pueden ser sospechadas cuando se aspira contenido intestinal, se presenta olor fecaloide o cuando la presión a la entrada se encuentra entre 8 y 10 milímetros de mercurio $(\mathrm{mm} \mathrm{Hg})$. En el posoperatorio inmediato debe ser considerada 
si la paciente presenta vómito, dolor abdominal, distensión y fiebre. ${ }^{5}$

\section{Lesiones urológicas}

Las lesiones de vejiga y uréteres se reportan con una incidencia de $0,03 \%$ a $0,13 \%$, donde las lesiones de la vejiga son de 2 a 3 veces más frecuentes que las lesiones ureterales. ${ }^{5}$ Los tipos de lesiones urinarias más comunes son perforación vesical, seguida de fístula, ligadura de uréter y corte de uréter; sin embargo, no se ha reportado diferencia con la cirugía abierta ginecológica. ${ }^{8}$ Los síntomas que pueden hacer sospechar lesión vesical son vómito, dolor o distensión abdominal, fiebre, escalofrío, retención urinaria, oliguria, anuria o goteo persistente de orina. ${ }^{5}$

\section{Complicaciones por el neumoperitoneo}

La disminución del retorno venoso, el aumento de la presión vascular pulmonar y sistémica, y la elevación de los niveles de dióxido de carbono son consecuencias fisiológicas del neumoperitoneo, al igual que la posición Trendelemburg, ambos requeridos durante los procedimientos ginecológicos laparoscópicos, los cuales son tolerados por la mayoría de las pacientes. Sin embargo, en mujeres con problemas cardiopulmonares puede ser causa de descompensación y de laparoconversión en pacientes no estabilizados. ${ }^{5}$ Por otra parte, casos de hipercapnia, neumotórax o neumomediastino y edema pulmonar han sido reportados, ${ }^{12-15}$ especialmente en pacientes con defectos del diafragma, usualmente en el hemidiafragma derecho y menos frecuente por perforación del diafragma durante los procedimientos laparoscópicos. ${ }^{8}$ Ocasionalmente, se puede presentar embolización con una frecuencia de $0,0014 \%$ y con una tasa de mortalidad de $28,5 \% .^{5,8}$ Otra complicación relacionada con el neumoperitoneo es el enfisema subcutáneo, que en la mayoría de los casos se limita a la pared abdominal, pero puede extenderse a extremidades, nuca, mediastino y pericardio. ${ }^{12}$

\section{Complicaciones según la técnica de entrada a la cavidad abdominal}

Revisaremos las complicaciones asociadas a la técnica cerrada (que utiliza la aguja de Veress), la técnica abierta (disección de la pared abdominal para el paso del trócar) y la técnica directa (paso directo del trócar sin uso de la aguja de Veress) ${ }^{16}$ así como la técnica visual. ${ }^{17}$

Se ha descrito que la entrada directa disminuye el riesgo de la insuflación preperitoneal y la falla de la entrada. ${ }^{5}$ Sin embargo, con esta técnica se presentan más lesiones viscerales en comparación con la aguja de Veress, pero menos lesiones vasculares ${ }^{18,19}$ y una incidencia más baja de complicaciones menores. ${ }^{20}$ No existe evidencia acerca de la superioridad de alguna técnica en relación a las otras. ${ }^{16,21,22}$ En una revisión de Cochrane en 2009 de 17 estudios controlados, en 3040 laparoscopias, no se presentaron diferencias entre las diversas técnicas para la prevención de las complicaciones mayores. Sin embargo, se presentaron 2 ventajas de la entrada directa, comparada con la aguja de Veress. Por un lado, la entrada directa evita la insuflación extraperitoneal (OR 0,06 IC 0,02-0,23) y, por otro lado, tiene una menor posibilidad de fracaso de la entrada (OR 0,22 IC 0,08-0,56). ${ }^{23}$

Por otra parte, no hay evidencia sobre una menor lesión durante el acceso mediante el uso de técnicas abiertas. En el año 2002, Molloy y colaboradores llevaron a cabo un metaanálisis, en el cual compararon la entrada directa, la entrada con aguja de Veress y la técnica abierta. En los resultados, se observó un mayor riesgo de lesiones intestinales con la técnica abierta (OR 2,9 IC $1,8-4,8)$. Por otra parte, se evidenció que la técnica abierta protege contra las lesiones vasculares cuando se compara con la técnica con aguja de Veress, con un OR de 0,1 (IC 0,01-0,8). ${ }^{16}$

En cuanto al sistema de cánula visual o trócares ópticos locales, el cual permite ir entrando a la cavidad y observar las diferentes capas de la pared abdominal, es preciso decir que no representa protección contra lesiones intraabdominales. ${ }^{17}$ 
En un ensayo aleatorizado con 186 pacientes posmenopáusicas, Tinelli y colaboradores no encontraron diferencias en la disminución de la ocurrencia de las complicaciones mayores vasculares e intestinales, cuando se usaron trócares ópticos, y solo presentó como ventaja una reducción en las complicaciones menores. ${ }^{24}$ Igualmente, en otro estudio aleatorizado, realizado por Minervini y colaboradores con 60 pacientes en procedimientos relacionados con patología renal, se llegó a la conclusión de que el uso de cánula visual no previene contra lesiones intraabdominales e incluso pueden ocurrir en un mayor porcentaje, cuando se compara con la técnica abierta de Hasson. ${ }^{25}$

Por otro lado, se han usado sitios diferentes para inserción de la aguja de Veress, cuando se sospechan adherencias a nivel periumbilical. El más usado es el denominado punto de Palmer, localizado a 3 centímetros por debajo del reborde costal con línea media clavicular. ${ }^{26}$ Esta entrada se encuentra contraindicada en pacientes con hepatoesplenomegalia o con antecedentes de cirugía gástrica o esplénica. ${ }^{26}$

\section{Hernias incisionales}

Tienen una incidencia aproximada de $1 \%,{ }^{8}$ con una variación entre $0,02 \%$ y $5 \% .{ }^{5}$ Ocurren con mayor frecuencia con la técnica cerrada en comparación con la abierta y están asociadas a factores como la obesidad, la desnutrición y la infección. ${ }^{27}$

Por otro lado, el riesgo de eventración aumenta con el diámetro del trócar. Es casi inexistente para trócares de $5 \mathrm{~mm}$, con un riesgo estimado de 0,23\% para trócares de $10 \mathrm{~mm}$, y 3,1\% para trócares de $12 \mathrm{~mm} .{ }^{28}$ La mayoría de las hernias ocurren en los sitios extraumbilicales y el uso de trócares de punta cónica disminuye la incidencia. ${ }^{8}$

\section{Otras complicaciones}

Las infecciones son raras, ${ }^{5,12}$ ya que se presentan en aproximadamente $0,1 \%$ de los casos. ${ }^{13}$ Otras complicaciones que se pueden presentar son los hematomas y la formación de adherencias; ${ }^{5}$ también se han descrito metástasis por el sitio del puerto, ${ }^{29}$ pero la incidencia no es diferente cuando se realiza laparotomía ${ }^{30}$ y no evita su uso en pacientes ginecológicas bien seleccionadas. ${ }^{31-33}$ Además, se pueden presentar lesiones neurológicas ocasionadas básicamente por mala posición de la paciente durante la cirugía. ${ }^{34}$

\section{Mortalidad}

En el año 2001, Bhoyrul y colaboradores analizaron 629 lesiones causadas por trócares, reportados a la Food and Drug Administration (FDA) entre 1993 y 1996. Se reportaron 32 muertes, donde 26 resultaron de injuria vascular y 6 de lesiones intestinales, en 28 muertes se usaron trócares desechables, 3 por visión óptica y 1 por trócares reutilizables, 408 lesiones fueron injurias vasculares y 182 lesiones viscerales, se presentaron 30 hematomas de pared y las demás pacientes no fueron analizadas. Estos autores concluyen que los trócares desechables y la entrada directa no disminuyen las complicaciones viscerales, y una lesión intestinal no reconocida puede ser fatal. ${ }^{35}$ Posteriormente, Fuller y colaboradores analizaron 1.399 lesiones por trócares entre 1997 y 2002 reportadas a la FDA. Se presentaron 31 muertes; 25 de ellas fueron por lesiones vasculares y 6 por lesiones intestinales; en 15 de las muertes se utilizaron trócares desechables, en 9 muertes se utilizaron trócares con visión óptica y 7 muertes no se reportaron. ${ }^{36}$ Por otro lado, en el metaanálisis llevado a cabo por Molloy y colaboradores se presentaron 10 muertes, en 5 casos no establecen como fue la entrada, en las otras 5 muertes reportadas se usó aguja de Veress, 2 fueron causadas por perforación intestinal y 3 relacionadas con embolismo gaseoso. Los autores no reportan el tipo de trócares que usaron. ${ }^{4}$

\section{CONCLUSIONES}

La laparoscopia es un procedimiento relativamente seguro, sin embargo hay que tener mucho cuidado 
durante el acceso a la cavidad abdominal porque la mayoría de complicaciones ocurren durante la entrada. Las nuevas tecnologías para la entrada en cirugía laparoscópica requieren mayor evaluación porque no hay evidencia que soporte un menor riesgo de lesiones vasculares e intestinales.

\section{AGRADECIMIENTOS}

A Lucy, mi compañera inseparable que me apoyó en esta nueva experiencia que comencé en mi vida; y a mi hija Andrea, motivo de mi impulso para la realización de mis metas.

\section{REFERENCIAS}

1. Morelli SS, McGovern PG. Laparoscopy in the gynecologic patient. Review of Techniques and Complications. Postgrad Obstet Gynecol 2009;29:1-5

2. Nordestgaard AG, Bodily KC, Osborne RW, Buttorff JD. Mayor vascular injuries during laparoscopic procedures. Am J Surg 1995;169:543-5.

3. Ahmad G, Duffy J, Watson A. Laparoscopic entry techniques and complications. Int J Gynaecol Obstet 2007;99:52-5.

4. Molloy D, Kaloo P, Cooper M, Nguyen T. Laparoscopic entry: a literature review and analysis of techniques and complications of primary port entry. Aust N Z J Obstet Gynaecol 2002;42:246-54.

5. Makai G, Isaacson K. Complications of gynecologic laparoscopy. Clin Obstet Gynecol 2009;52:401-11.

6. Tarik A, Fehmi C. Complications of gynaecological laparoscopy--a retrospective analysis of 3572 cases from a single institute. J Obstet Gynaecol 2004;24:813-6.

7. Chapron C, Fauconnier A, Goffinet F, Bréart G, Dubuisson J. Laparoscopic surgery is not inherently dangerous for patients presenting with benign gynecologic pathology. Results of a meta-analysis. Hum Reprod 2002;17:1334-42.

8. Magrina J. Complications of laparoscopic surgery. Clin Obstet Gynecol 2002;45:469-80.

9. Shirk GJ, Johns A, Redwine DB. Complications of laparoscopic surgery: How to avoid them and how to repair them. J Minim Invasive Gynecol 2006;13:352-9.

10. Vázquez-Frías JA, Huete-Echandi F, Cueto-García J, Padilla-Paz LA. Prevention and treatment of abdominal wall bleeding complications at trocar sites: review of the literature. Surg Laparosc Endosc Percutan Tech 2009;19:195-7.

11. Stovall TG, Mann WJ. Complications of gynecologic laparoscopic surgery. Up To Date 2009;17(1).

12. Munro MG. Laparoscopic access: complications, technologies, and techniques. Curr Opin Obstet Gynecol 2002;14:36-74.

13. Wadlund DL. Laparoscopy: risks, benefits and complications. Nurs Clin N Am 2006;41:219-29.

14. Gutiérrez MA, Navarro, Cardoso B, Garzon JP. Edema Pulmonar en Cirugía laparoscópica ginecológica. Rev Col Anest 2007; 35:167-70.

15. Eslava J, Gaitán H, Pedraza N. Edema pulmonar en cirugía laparoscópica: presentación de un caso y revisión sistemática de la literatura. Rev Colomb Obstet Ginecol 2005;56:294-302.

16. Vilos GA, Ternamian A, Dempster J, Laberge PY. The Society of Obstetricians and Gynaecologists of Canada. Laparoscopic entry: a review of techniques, technologies, and complications. J Obstet Gynaecol Can 2007;29:433-65.

17. Jansen FW, Kolkman W, Bakkum EA, de Kroon CD, Trimbos-Kemper TC, Trimbos JB. Complications of laparoscopy: an inquiry about closed versus open-entry technique. Am J Obstet Gynecol 2004;190;634-8.

18. Chapron C, Cravello L, Chopin N, Kreiker G, Blanc B, Dubuisson JB. Complications during set-up procedures for laparoscopy in gynecology: open laparoscopy does not reduce the risk of major complications. Acta Obstet Gynecol Scand 2003;82:1125-9.

19. Royal College of Obstetricians and Gynaecologists. Preventing Entry-Related Gynaecological Laparoscopic Injuries Green-top Guideline No. 49 May 2008.

20. Chandler JG, Corson SL, Way L. Three spectra of laparoscopic entry access injuries. J Am Coll Surg 2001;192:478-91.

21. Vilos GA. The ABCs of a safer laparoscopic entry. J Minim Invasive Gynecol 2006;13:249-51.

22. Ahmad G, Duffy JMN, Phillips K, Watson AJS. Laparoscopic Entry Techniques. Cochrane Database Syst Rev 2008;(2):CD006583.

23. Sharp HT, Dodson MK, Draper ML, Watts DA, Doucette RC, Hurd WW. Complications associated with optical-access laparoscopic trocars. Obstet Gynecol 2002;99:553-5. 
24. Tinelli A, Malvasi A, Guido M, Istre O, Keckstein J, Mettler L. Initial laparoscopic access in postmenopausal women: a preliminary prospective study. Menopause 2009; 16:966-70.

25. Minervini A, Davenport K, Pefanis G, Keeley FX Jr, Timoney AG. Prospective study comparing the bladeless optical access trocar versus hasson open trocar for the establishment of pneumoperitoneum in laparoscopic renal procedures. Arch Ital Urol Androl 2008;80:95-8.

26. Brill AI, Cohen BM. Fundamentals of Peritoneal Access. J Am Assoc Gynecol Laparosc 2003;10:287-97.

27. Tonouchi H, Ohmori Y, Kobayashi M, Kusunoki M. Trocar site hernia. Arch Surg 2004;139:1248-56.

28. Leroy J, Dutson E, Henri M. Complicaciones vinculadas a las puertas de acceso y trócar. WeBSurg. com 2005;5(3). Visitado 2009 Oct 10. Disponible en: http://www.websurg.com/ref/doi-ot02es274.htm

29. Ramirez PT, Frumovitz M, Wolf JK, Levenback C. Laparoscopic port-site metastases in patients with gynecological malignancies. Int J Gynecol Cancer 2004;14:1070-7.

30. Vergote I, Marquette S, Amant F, Berteloot P, Neven P. Port-site metastases after open laparoscopy: a study in 173 patients with advanced ovarian carcinoma. Int J Gynecol Cancer 2005;15:776-9.

31. Zivanovic O, Sonoda Y, Diaz JP, Levine DA, Brown CL, Chi DS, et al. The rate of port-site metastases after 2251 laparoscopic procedures in women with underlying malignant disease. Gynecol Oncol 2008;111:431-7.

32. Abu-Rustum NR, Rhee EH, Chi DS, Sonoda Y, Gemignani M, Barakat RR. Subcutaneous tumor implantation after laparoscopic procedures in women with malignant disease. Obstet Gynecol 2004;103:480-7.

33. Curet MJ. Port site metastases. Am J Surg 2004;187:705-12.

34. Barnett JC, Hurd WW, Rogers RM, Williams NL, Shapiro SA. Laparoscopic positioning and nerve injuries J Min Invasive Gynecol 2007:14:664-72.

35. Bhoyrul S, Vierra MA, Nezhat CR, Krummel TM, Way LW. Trocar injuries in laparoscopic surgery. J Am Coll Surg 2001;192:677-83.

36. Fuller J, Ashar BS, Carey-Corrado J. Trocarassociated injuries and fatalities: An analysis of 1399 reports to the FDA. J Minim Invasive Gynecol 2005;12:302-7. 